\title{
DESARROLLO SOSTENIBLE EN TURISMO: Una propuesta para Machu Picchu*
}

\author{
Otto Regalado Pezúa \\ UNIVERSIDAD ESAN \\ oregalado@esan.edu.pe
}

\section{Jesús Arias Valencia}

ESPECIALISTA EN GESTIÓN AMBIENTAL Sostenible

canaria@terra.com.pe

\section{Resumen}

Este artículo persigue tres objetivos: el primero, crear conciencia sobre los riesgos ambientales que amenazan al Santuario Histórico de Machu Picchu (SHM); el segundo, brindar elementos de reflexión que contribuyan a resolver la problemática del SHM; y finalmente, enriquecer el debate presentando una propuesta para la gestión sostenible del SHM. Para ello, en la primera sección se muestran cifras que evidencian la unipolaridad del Perú como destino turístico; luego, en la segunda sección, se expone la compleja problemática identificada en el SHM; y, para terminar, se presenta la propuesta de solución, que pasa por plantear la creación de una estructura de gestión que se encargue de la planificación estratégica del SHM respetando los principios clave del desarrollo sostenible.

Palabras clave: impacto ambiental, destino unipolar, área natural protegida, patrimonio cultural y natural, capacidad de carga, unidad de gestión, plan maestro, desarrollo sostenible, turismo alternativo, Machu Picchu.

\begin{abstract}
Raising awareness about the environmental risks faced by the Historical Sanctuary of Machu Picchu, providing tools for thinking that will contribute to addressing the Sanctuary's problems, and finally, contributing to the debate by forwarding a proposal for the Sanctuary's sustainable management are the purposes of this study. In the first section, statistics show Peru is basically a unipolar travel destination. The second section presents the complex problems identified at the Sanctuary. To conclude, the study presents a proposed solution, including setting up a structure to manage the Sanctuary's strategic planning while abiding by the key principles of sustainable development.
\end{abstract}

Key words: environmental impact, unipolar destination, protected natural area, cultural and natural heritage, carrying capacity, management unit, master plan, sustainable development, alternative tourism, Machu Picchu.

* Una versión anterior de este trabajo, con el título «Sustainable development in tourism: a proposition for Machupicchu, Peru», ha sido incluida en: Managing world heritage sites, compilación de los profesores Anna Leask y Alan Fyall, de Napier University y Bournemouth University, respectivamente, ambas de Edimburgo, Escocia. El libro ha sido publicado este año por la editorial europea Elsevier. 


\section{Introducción}

A pesar de su vasta geografía y de la enorme variedad de prácticas turísticas que puede acoger en su territorio, el Perú sigue posicionado como un destino turístico unipolar. Los visitantes se concentran en la ciudad del Cusco y en el llamado «circuito sur», cuya principal atracción es el Santuario Histórico de Machu Picchu (SHM). De allí la importancia de la preservación, conservación y planificación del desarrollo de este patrimonio cultural y natural de la humanidad.

No obstante, las condiciones actuales en las que se desenvuelve la actividad turística en el SHM no son las mejores; estudios recientes han puesto sobre el tapete los riesgos que corren las ruinas y sus alrededores. Esta situación refleja la importancia del papel de los gobiernos respecto del desarrollo turístico. Por un lado, las políticas adecuadas pueden aportar grandes ventajas y beneficios en los ámbitos económico, educativo y sociocultural; pero, por otro lado, las políticas desacertadas pueden contribuir a la degradación ambiental del sitio turístico y a la pérdida de identidad de la población local, entre otros efectos negativos.

El presente estudio de caso orienta al lector sobre la dimensión del turismo en el Perú y sus principales centros de interés, luego describe la problemática -causas y consecuencias- del Santuario Histórico de Machu Picchu como destino turístico natural y cultural y, finalmente, presenta una alternativa de solución basada en los principios clave del desarrollo sostenible.

\section{Cifras del turismo en el Perú}

Diversos estudios coinciden en que el Perú es percibido como un destino turístico histórico, arqueológico y cultural. La Comisión de Promoción del Perú (PromPerú) indica que la ciudad del Cusco, el SHM y/o el «circuito sur» son visitados por el $50 \%$ de los turistas que ingresan al país, mientras el informe de Monitor Company, más preciso, señaló que entre el $70 \%$ y el $75 \%$ de los turistas que llegan al Perú visitan la ciudad del Cusco y el SHM. La definición de los conceptos de «turista»y «visitante» es clave para la comprensión de estas cifras. Sin embargo, lo evidente es el posicionamiento que tiene nuestro país como destino turístico unipolar, pues, según el modelo turístico de Leiper, la ciudad de Lima constituye una zona de tránsito.

Las diversas fuentes señalan la gran variación en los registros de visitantes, incluso en el caso de la información proporcionada por los organismos oficiales. La Dirección General de Migraciones y Naturalización (Digemin) indica que en el año 2005 llegaron al Perú 1486005 visitantes internacionales. El Instituto Nacional de Cultura (INC) señala que de los 679951 turistas que visitaron el SHM durante ese mismo año, 204636 (30\%) fueron nacionales y $475315(70 \%)$ extranjeros.

La figura 1, elaborada en el año 2004 por PromPerú, muestra los principales destinos visitados en el Perú durante el año 2003 y el porcentaje de visitantes recibidos por cada uno (sobre el total). Según PromPerú, si bien los turistas visitaron Lima, la mayoría de ellos tuvo como destino principal la ciudad del Cusco, el SHM y el Valle Sagrado (ubicado a $60 \mathrm{~km}$ del 


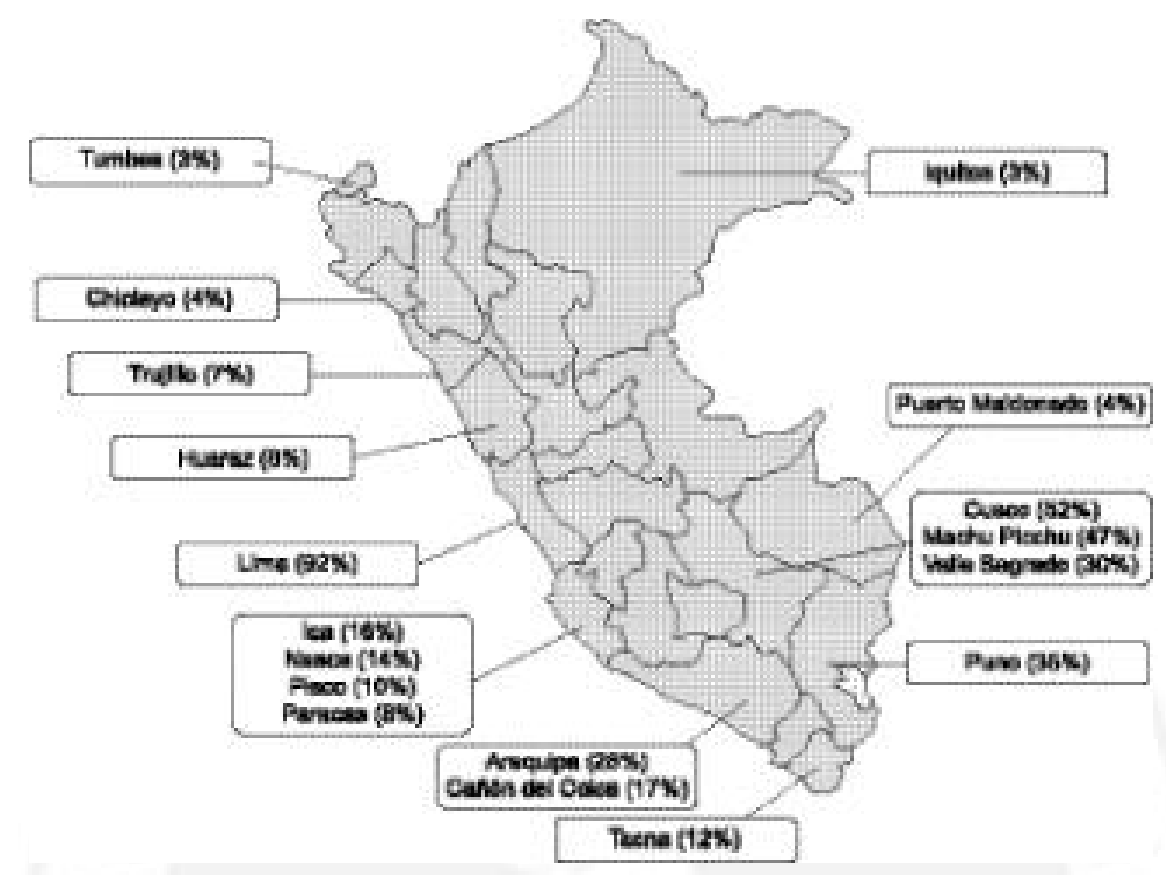

Fuente: PromPerú (2004: pág. 9).

Figura 1

Principales destinos visitados en el Perú

Cusco), y luego viajaron a otros destinos de la región sur, como Puno, Arequipa, Ica y Tacna.

Estas cifras bastan para comprender el papel que cumple el SHM como principal destino turístico del Perú y el correspondiente interés en su cuidado y preservación. A continuación presentamos una breve reseña sobre el SHM y sus riquezas naturales y culturales, así como la problemática que generan las actividades turísticas y agropecuarias administradas sin una unidad de gestión adecuada.

\section{EI Santuario Histórico de Machu Picchu (SHM)}

La ciudad inca de Machu Picchu fue redescubierta y revelada a la ciencia por el historiador y explorador norteamericano Hiram Bingham en 1911, pero recién a partir de 1950 se iniciaron las actividades turísticas y, por lo tanto, el desarrollo de la infraestructura y servicios necesarios para atender a los visitantes. A comienzos de los años ochenta se observó un rápido crecimiento del turismo hacia esta joya arqueológica; sin embargo, debido a la subversión terrorista y a los problemas económicos que el Perú atravesaba, la actividad decayó notoriamente y no se reactivó hasta 1992, cuando cesó la violencia social. Desde entonces ha mantenido una tendencia creciente.

El SHM, ubicado en un área natural protegida de 32592 hectáreas, fue declarado Patrimonio Cultural y Natural de la Humanidad por la Unesco en 1983. Esta 
privilegiada zona ha llamado la atención por el conjunto de sus valores culturales: la antigua ciudad inca de Machu Picchu, la red de caminos inca, y más de 35 sitios arqueológicos distribuidos en un entorno natural sobresaliente. El conjunto es depositario de una notable biodiversidad. Según estudios realizados en los últimos años, las especies de flora y fauna registradas representan una alta proporción con respecto al resto del país; además se observa la presencia de especies de distribución restringida, algunas de ellas con alto riesgo de extinción (entre $10 \%$ y 20\%).

El crecimiento mundial del turismo llamado de naturaleza y cultura origina mayor interés por visitar el SHM. Como hemos señalado anteriormente, las entradas registradas durante el 2005 dan cuenta de más de 600 mil visitantes y se estima que más de 12 millones de personas ya han sido atraídas por este enigmático lugar. Es evidente que la presencia creciente de visitantes ha aumentado el impacto sobre el ambiente: mayor generación de residuos sólidos; procesos de urbanización; uso directo y no controlado de los espacios que presentan mayor atractivo cultural y natural, principalmente la ciudad inca, la red de caminos inca y algunos de sus bosques primarios. Es importante notar que la cuenca del río Vilcanota (denominado también río Urubamba), que atraviesa el SHM, recibe los residuos y las aguas negras del mayor conglomerado humano de la región.

Esta situación, sumada a la vulnerabilidad natural de los ecosistemas, ha desencadenado procesos de deterioro que amenazan la persistencia y el potencial de disfrute futuro de los valores naturales y culturales del SHM. Ante este hecho, la
Unesco, en coordinación con las autoridades peruanas, ha solicitado la realización de diversos estudios de evaluación y monitoreo sobre el estado actual de los recursos albergados en esta área protegida.

Pero no sólo las actividades turísticas ponen en riesgo el ecosistema de Machu Picchu. Las labores agropecuarias realizadas en el interior del santuario han ocasionado incendios forestales, deforestación -debido a la necesidad de combustible (leña) - y la degradación de los suelos por el sobrepastoreo, entre otros daños.

De la revisión y análisis de los informes emitidos por diferentes misiones de evaluación de la Unesco y de otros documentos de diversas entidades públicas y privadas que han trabajado y continúan trabajando en la zona, hemos identificado la problemática que resumimos en el cuadro de la página siguiente.

A fin de solucionar estos problemas, así como sus causas y efectos, es determinante la participación activa de los niveles más altos de la administración pública nacional en el aspecto estratégico, y de los actores locales en el ámbito operativo. Sólo cuando haya la voluntad política que priorice los objetivos de conservación del SHM y se logre la participación de la comunidad local, podremos pensar en el desarrollo turístico sostenible de este importante legado arqueológico.

El diseño y las aplicaciones de estas estrategias deberán ser compatibles con los tres principios fundamentales del desarrollo sostenible: la rentabilidad económica de largo plazo, la conservación y aprovechamiento racional de los recursos turísticos naturales y la participación de la población local. 


\section{Problemática identificada en el Santuario Histórico de Machu Picchu}

\begin{tabular}{|c|c|c|}
\hline Problemas & Causas & Efectos \\
\hline \multirow{3}{*}{$\begin{array}{l}\text { Conflictos } \\
\text { generados por } \\
\text { actividades } \\
\text { turísticas }\end{array}$} & $\begin{array}{l}\text { No se comprende el concep- } \\
\text { to de «gestión de capacidad } \\
\text { de carga», por lo que este } \\
\text { aún no es incorporado en los } \\
\text { procesos de planificación. }\end{array}$ & $\begin{array}{l}\text { Sobrecarga de visitantes durante } \\
\text { los recorridos en la ciudad inca y } \\
\text { en la red de caminos inca, } \\
\text { especialmente en temporada alta, } \\
\text { genera grandes deterioros. } \\
\text { Crecimiento no planificado de los } \\
\text { turistas (gran afluencia estacional } \\
\text { de visitantes). }\end{array}$ \\
\hline & $\begin{array}{l}\text { - Gran demanda de servicios } \\
\text { turísticos (transporte, } \\
\text { alojamiento, alimentación, } \\
\text { entre otros). }\end{array}$ & $\begin{array}{l}\text { - Crecimiento urbano desordenado } \\
\text { en los puntos de acceso al SHM } \\
\text { y en puntos intermedios al interior } \\
\text { del área natural (Aguas Calientes). } \\
\text { - Invasión de terrenos, en donde se } \\
\text { desarrolla infraestructura precaria } \\
\text { de servicios. } \\
\text { - Falta de adecuados sistemas de } \\
\text { gestión de residuos sólidos y de } \\
\text { aguas negras. } \\
\text { - Servicios turísticos de muy baja } \\
\text { calidad. } \\
\text { - Desarrollo de infraestructura } \\
\text { hotelera. } \\
\text { - Crecimiento urbano. } \\
\text { - Desarrollo de servicios públicos de } \\
\text { transporte (ferrocarril, helicópteros } \\
\text { y ómnibus). } \\
\text { - Demanda de animales de carga } \\
\text { (caballos, mulas y burros). }\end{array}$ \\
\hline & $\begin{array}{l}\text { No se realizan adecuados } \\
\text { procesos de planificación } \\
\text { estratégica ni operativa (plan } \\
\text { maestro, planes de sitio, } \\
\text { planes anuales) para los } \\
\text { diversos atractivos. }\end{array}$ & $\begin{array}{l}\text { - Sobreposición de jurisdicciones y } \\
\text { responsabilidades institucionales. } \\
\text { - Desorganización y caos institucio- } \\
\text { nal al momento de implementar los } \\
\text { planes operativos individuales. } \\
\text { - Uso irracional de la tierra y falta de } \\
\text { su discriminación por sectores } \\
\text { (necesidad de una microzonifica- } \\
\text { ción). }\end{array}$ \\
\hline
\end{tabular}




\begin{tabular}{|c|c|c|}
\hline Problemas & Causas & Efectos \\
\hline \multirow[t]{2}{*}{$\begin{array}{c}\text { Conflictos generados } \\
\text { por actividades } \\
\text { agrícolas y } \\
\text { pecuarias }\end{array}$} & $\begin{array}{l}\text { - Actividades agropecuarias } \\
\text { incompatibles con el SHM. } \\
\text { - Limitada capacidad de } \\
\text { monitoreo, por parte de las } \\
\text { autoridades, de las activida- } \\
\text { des que se desarrollan en } \\
\text { el SHM. }\end{array}$ & $\begin{array}{l}\text { - No se cumple la normatividad } \\
\text { vigente. } \\
\text { - Incendios forestales. } \\
\text { - Disminución de la diversidad } \\
\text { arbórea y deforestación. } \\
\text { - Demanda de combustibles (leña). } \\
\text { - Introducción de diversas especies } \\
\text { exóticas que compiten y despla- } \\
\text { zan a las especies nativas. } \\
\text { - Ganadería doméstica de animales } \\
\text { exóticos. } \\
\text { - Erosión y degradación de las } \\
\text { zonas de pastoreo (capacidad de } \\
\text { carga superada hasta en cinco } \\
\text { veces). } \\
\text { - Caza furtiva y extracción de } \\
\text { especies de flora. } \\
\text { - Pérdida de biodiversidad. }\end{array}$ \\
\hline & $\begin{array}{l}\text { - Falta de planificación en el } \\
\text { desarrollo de actividades } \\
\text { agropecuarias. }\end{array}$ & $\begin{array}{l}\text { - A pesar de las propuestas } \\
\text { existentes, aún no se implementa } \\
\text { la zonificación de área natural } \\
\text { protegida. }\end{array}$ \\
\hline $\begin{array}{l}\text { Conflictos generados } \\
\text { por servicios } \\
\text { públicos }\end{array}$ & $\begin{array}{l}\text { - Ausencia de planificación } \\
\text { en el desarrollo de activida- } \\
\text { des relacionadas con los } \\
\text { servicios públicos. } \\
\text { - Limitada normatividad } \\
\text { ambiental para la regulación } \\
\text { de servicios públicos dentro } \\
\text { del SHM. } \\
\text { - Limitada capacidad de } \\
\text { monitoreo de las actividades } \\
\text { económicas y sociales } \\
\text { complementarias al interior } \\
\text { y fuera del SHM. }\end{array}$ & $\begin{array}{l}\text { - Ampliaciones y refacciones en la } \\
\text { central de generación hidroeléctri- } \\
\text { ca sin estudios adecuados de } \\
\text { impacto ambiental. } \\
\text { - Inexistente monitoreo ambiental } \\
\text { de los servicios de transporte } \\
\text { público al interior y fuera del SHM. } \\
\text { - Ausencia de protocolos ambienta- } \\
\text { les de operación. } \\
\text { - Derrames de combustible, } \\
\text { emisión de ruidos contaminantes } \\
\text { y de gases de combustión } \\
\text { incompleta. } \\
\text { - Talleres de maestranza y repara- } \\
\text { ción precarios (infraestructura } \\
\text { física inapropiada). } \\
\text { - Ausencia de estudios de impacto } \\
\text { ambiental (ESIA) y evaluaciones } \\
\text { de impacto ambiental (EIA) en las } \\
\text { actividades económicas y } \\
\text { sociales. }\end{array}$ \\
\hline
\end{tabular}




\section{Propuesta de solución}

Por lo expuesto hasta aquí, creemos que el principal factor crítico de éxito en la búsqueda de soluciones está relacionado con mecanismos de participación de los diferentes actores y grupos de interés en los procesos de planificación estratégica y ejecución de programas. Estos mecanismos deberán establecer lineamientos y restricciones en el desarrollo de dichos procesos y no solamente «consensos» en las negociaciones que satisfagan únicamente los intereses de individuos e instituciones particulares y dejen de lado los objetivos de conservación del SHM.

Asimismo, la coexistencia de diversas entidades públicas con jurisdicción legal sobre el SHM dificulta la priorización de actividades para su gestión e, involuntariamente, agudiza la problemática.

Ante esta situación, nuestra propuesta para lograr el desarrollo sostenible del SHM requiere la creación de una unidad de gestión que permita agilizar el saneamiento legal de este patrimonio e iniciar el proceso de valoración de los servicios ambientales. Todo ello integrado en un contexto de desarrollo regional.

Para tal fin, las autoridades nacionales deberán formar una entidad autónoma (técnica, económica y administrativa) que incluya un directorio internacional como instancia de mayor nivel de decisión sobre el manejo del SHM. Este directorio deberá estar a cargo de un director ejecutivo e integrado por representantes del sector público nacional, de organismos de conservación, del sector privado nacional y de instituciones internacionales especializadas.
Como unidad de apoyo, el directorio deberá contar con un consejo asesor integrado por personalidades, nacionales e internacionales, provenientes de diversos campos científicos y tecnológicos que contribuyan al desarrollo sostenible del SHM en sus diferentes especialidades.

Como unidad de acción proponemos la formación de un comité de gestión integrado por representantes de las diferentes entidades públicas y privadas que actúan en el ámbito de influencia del SHM. Su principal función será facilitar y apoyar los procesos de planificación y gestión de riesgos de las estrategias identificadas para el SHM.

Hemos identificado seis estrategias generales para alcanzar los objetivos establecidos por el Plan Maestro del SHM vigente, el mismo que deberá ser actualizado y adecuado metodológicamente a la planificación estratégica con un enfoque de gestión de riesgos.

En la figura 2 presentamos un esquema que resume nuestra propuesta para la gestión turística sostenible del SHM.

Como hemos señalado anteriormente, la puesta en marcha de esta estructura de gestión favorecerá la actualización constante del Plan Maestro del SHM. El objetivo principal será establecer lineamientos que permitan el ordenamiento del uso turístico de la zona, el mejoramiento de la calidad de vida de la población local, así como la preservación de la capacidad de carga ecológica y la obtención de mayores beneficios; todo ello garantizará el manejo concertado del patrimonio natural y cultural del SHM. 


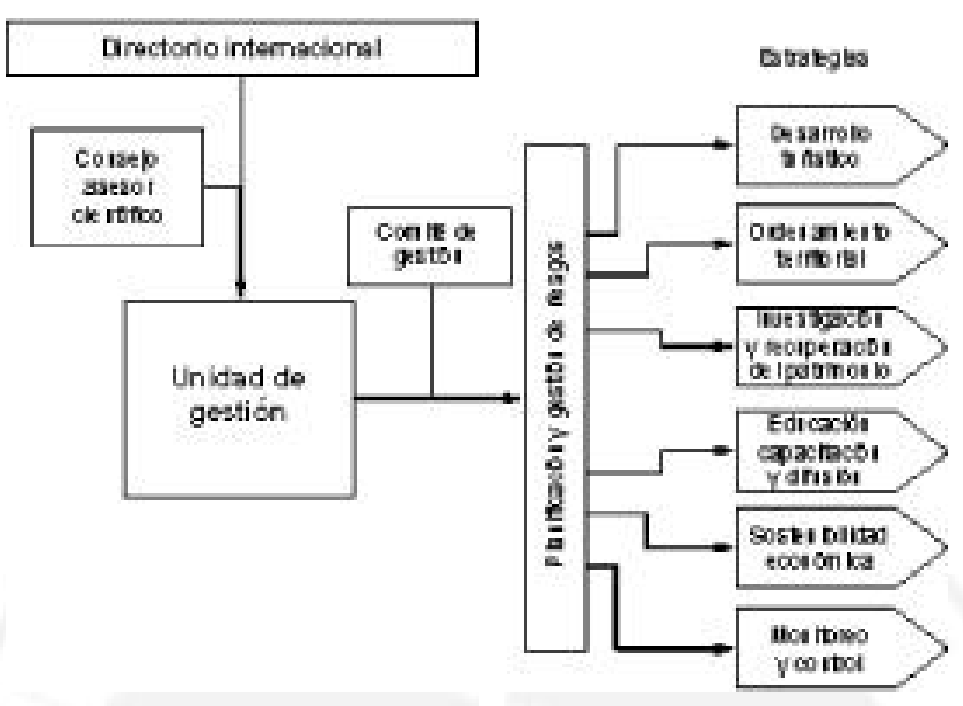

Figura 2

Estructura de gestión propuesta para el SHM

La estrategia vinculada al desarrollo turístico de la zona permitirá la planificación de las actividades turísticas. Comprenderá lo siguiente: prever la satisfacción de los turistas-consumidores, garantizar la calidad de servicio acorde con estándares preestablecidos, controlar el acceso de turistas según la capacidad de carga máxima de visitantes, diversificar la oferta de servicios y prever futuras necesidades de acuerdo con la sostenibilidad del medio ambiente.

La estrategia concerniente al ordenamiento territorial permitirá resolver el saneamiento físico y legal del SHM. Asimismo, hará posible evaluar la demarcación y ampliación de sus límites territoriales. Por otro lado, esta estrategia permitirá aplicar correctamente los criterios de sostenibilidad a todas las actividades económicas y socioculturales del SHM, en los ámbitos rural y urbano.
La estrategia relativa a la investigación y recuperación del patrimonio permitirá la evaluación, programación y ejecución de acciones de restauración y conservación del patrimonio natural y cultural del SHM. Esta planificación deberá apoyarse en investigaciones científicas aceptadas y aprobadas por entidades competentes.

La estrategia de educación, capacitación y difusión estará dirigida a transmitir la sensibilidad medioambiental a todas las personas involucradas, directa e indirectamente, con el SHM. La implementación de esta estrategia permitirá a la población local involucrarse en programas participativos vinculados a las labores de conservación y comprometerá a los visitantes con la preservación del SHM.

La estrategia de sostenibilidad económica, como su nombre lo indica, buscará beneficiar económicamente a la población 
local creando puestos de trabajo y aumentando los ingresos de los trabajadores, pero respetando los indicadores de capacidad de carga relacionados con la inversión, la infraestructura suficiente y el equipamiento necesario.

Finalmente, la estrategia relacionada con el monitoreo y control propondrá la implementación de un sistema de monitoreo integral que permita el control preventivo y la mitigación de impactos ambientales negativos sobre el SHM, estos últimos generados por las actividades económicas y socioculturales que se llevan a cabo en la zona.

Todas estas estrategias interactúan entre sí, por lo que deberán ser tratadas de forma integral.

\section{Conclusiones}

Diversos estudios coinciden en que el Perú es percibido como un destino turístico histórico, arqueológico y cultural. Cifras de diversas fuentes señalan que entre el 50 y el $75 \%$ de los turistas extranjeros visitan la ciudad del Cusco y el SHM, lo que califica al país como un destino turístico unipolar. Sin embargo, las condiciones en las que se desarrolla la actividad turística en esta zona ponen en riesgo el SHM, patrimonio histórico, cultural y natural de la humanidad.

A pesar de ser el principal destino turístico del Perú, el SHM no es administrado con una gestión que garantice su sostenibilidad. Todo lo contrario, las medidas adoptadas de manera aislada sólo contribuyen al deterioro ambiental. No únicamente el turismo desproporcionado genera problemas ambientales al SHM, sino también las labores agrícolas y pecuarias de la población local y las actividades conexas a la actividad turística, como son la infraestructura inapropiada o la urbanización no planificada.

Nuestra propuesta para la sostenibilidad turística del SHM se inscribe en los principios clave del desarrollo sostenible. Así, proponemos la creación de una estructura de gestión encargada de la planificación estratégica del SHM que cuente con un directorio internacional y un consejo asesor científico, integrados ambos por personalidades competentes.

Paralelamente, proponemos la formación de un comité de gestión encargado de facilitar y apoyar los procesos de planificación y gestión de riesgos en la implementación de las estrategias identificadas: estrategias de desarrollo turístico; de ordenamiento territorial; de investigación y recuperación del patrimonio; de educación, capacitación y difusión; de sostenibilidad económica; y de monitoreo y control. Estas estrategias deberán ser tratadas de forma integral debido a que están estrechamente relacionadas e interactúan entre sí.

\section{Perspectivas}

Si continúan aumentando los impactos negativos sobre el ambiente, podría disminuir el volumen de visitas al SHM. Esta coyuntura obligaría a las autoridades a tomar decisiones extremas, como cerrar parcial o completamente el SHM por un determinado periodo, en aras de su recuperación y relanzamiento turístico. En busca de una sostenibilidad turística para el SHM, conviene hacerse las siguientes preguntas: 
- ¿Qué sucedería con la actividad turística del país y cómo se vería afectada la economía de la región?

- ¿Podrían los otros destinos turísticos del país reemplazar la oferta turística del SHM? ¿La demanda estaría interesada en dichos destinos?
- ¿Cuánto tiempo más el patrimonio albergado en el SHM puede seguir soportando el estilo actual de su gestión? ¿Qué medidas deben tomarse para evitar que los daños sean científica y técnicamente irreversibles?

\section{Referencias bibliográficas}

COMISIÓN DE PROMOCIÓN DEL PERÚ (PromPerú). 2004. Perfil del turista extranjero 2003. Lima: PromPerú.

INRENA-INC. Plan Maestro del Santuario Histórico de Machupicchu. Aprobado por Resolución Jefatural n. ${ }^{\circ}$ 085-98-INRENA, del 22 de octubre de 1998.

—. Actualización del Plan Maestro de Machupicchu. Aprobado por Resolución Jefatural $n .^{\circ}$ 109-2005-INRENA, del 1 de junio de 2005 .

LEIPER, N. 2004. Tourism management. $3^{\text {rd }}$ ed. Frenchs Forest, New South Wales (Australia): Pearson Education.

LOZATO-GIOTART, J. P. 2003. Géographie du tourisme: De l'espace consommé à l'espace maîtrisé. Paris: Pearson Education, Collection Tourisme.

MONITOR COMPANY. 1995. Construyendo las ventajas competitivas del Perú. El turismo. Lima: PromPerú.

PERÚ. CONSEJO NACIONAL DEL AMBIENTE (Conam). 2003. Lineamientos para la promoción del turismo sostenible en las regiones. Aprobados por Consejo Directivo. Sesión del día 28 de agosto de 2003. Decreto del Consejo Directivo . $^{\circ}$ 039-2003-CONAM/PCD. Consejo Nacional del Ambiente.

PROFONANPE. 2002. Ampliación de la base de datos sobre diversidad biológica del Santuario Histórico de Machu Picchu: Diversidad botánica. Informe final. Instituto de Investigaciones en Ciencias Biológicas de la Universidad de San Antonio Abad del Cusco.

- 2002. Ampliación de la base de datos sobre diversidad biológica del Santuario Histórico de Machu Picchu: Diversidad zoológica. Informe final. Asociación para la Conservación de la Selva Sur y del Museo de Historia Natural de la Universidad de San Antonio Abad del Cusco.

—. 2002. Biodiversidad del Santuario Histórico de Machu Picchu: estado actual del conocimiento. Centro de Datos para la Conservación, de la Universidad Nacional Agraria La Molina, Lima. 
REGALADO-PEZÚA, O. y ARIAS-VALENCIA, J. 2004. Posicionamiento de Machu Picchu en el sistema turístico del Perú: ¿riesgo u oportunidad? Ponencia presentada al seminario: «Between Sustainable Tourism and Local Development: Prospects and Paradoxes». Centro de Estudios y Documentación Latinoamericanos (Cedla), Amsterdam, 8-10 de diciembre.
REGALADO-PEZUA O. y FERRY, M. 2005.

De l'optimum touristique à l'optimum marketing: une proposition pour le Machu Picchu. Journée thématique de recherché en Marketing du tourisme et de loisirs. Institut de Recherche en Gestion et Economie de l'Université de Savoie. Annecy, le 10 juin. 